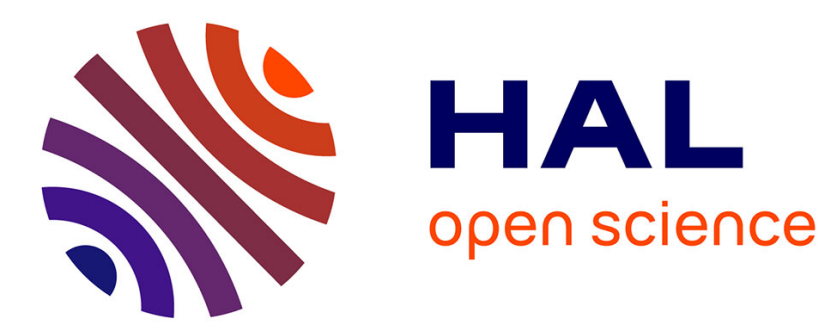

\title{
Liquid spreading on cold surfaces: Solidification-induced stick-slip dynamics
}

Rémy Herbaut, Philippe Brunet, Laurent Limat, Laurent Royon

\section{To cite this version:}

Rémy Herbaut, Philippe Brunet, Laurent Limat, Laurent Royon. Liquid spreading on cold surfaces: Solidification-induced stick-slip dynamics. Physical Review Fluids, 2019, 4 (3), 10.1103/PhysRevFluids.4.033603 . hal-02343306

\section{HAL Id: hal-02343306 https://hal.science/hal-02343306}

Submitted on 8 Nov 2019

HAL is a multi-disciplinary open access archive for the deposit and dissemination of scientific research documents, whether they are published or not. The documents may come from teaching and research institutions in France or abroad, or from public or private research centers.
L'archive ouverte pluridisciplinaire HAL, est destinée au dépôt et à la diffusion de documents scientifiques de niveau recherche, publiés ou non, émanant des établissements d'enseignement et de recherche français ou étrangers, des laboratoires publics ou privés. 


\title{
Liquid spreading on cold surfaces: Solidification-induced stick-slip dynamics
}

\author{
Rémy Herbaut, ${ }^{1}$ Philippe Brunet, ${ }^{1}$ Laurent Limat, ${ }^{1}$ and Laurent Royon ${ }^{2}$ \\ ${ }^{1}$ Laboratoire Matière et Systèmes Complexes, Université Paris Diderot, \\ UMR 7057 CNRS, F-75013 Paris, France \\ ${ }^{2}$ Laboratoire Interdisciplinaire des Energies de Demain, Université Paris Diderot, \\ UMR 8236 CNRS, F-75013 Paris, France
}

(Received 1 August 2018; published 27 March 2019)

\begin{abstract}
We study the contact line dynamics of a liquid drop on a substrate at temperature $T_{s}$ colder than the freezing temperature $T_{m}$. The purpose is to determine experimentally the criterion for a solidification-induced pinning of the liquid at its triple line. The substrate is driven at constant velocity, while the upper part of the drop is pinned to the injection pipe. Within a certain range of substrate velocity and temperature difference $T_{m}-T_{s}$, the solidification can induce contact-line pinning. This pinning occurs when the velocity of the substrate is slower than a critical value $V_{c}$ and leads to a discontinuous stick-slip dynamics of the contact line, while at high enough velocity the contact-line motion remains continuous. Stick-slip dynamics appear when the solid front, showing dendritic structure, catches up the advancing contact line. We study the dynamics of the stick-slip regime at different substrate velocities $V_{s}, T_{m}-T_{s}$, and injection flow rate $Q$. We relate the dependence of $V_{c}$ on $T_{m}-T_{s}$ to the velocity of the solidification front $V_{f}$ calculated and measured with an independent experiment. These results are consistent with a mechanism of kinetic undercooling where the front velocity follows a trend reminiscent to that measured and predicted in dendritic solidification front growth.
\end{abstract}

DOI: 10.1103/PhysRevFluids.4.033603

\section{INTRODUCTION}

How does solidification influence the dynamics of liquid spreading on a substrate, and what determines the arrest of spreading by the solid under formation? This fundamental question is of primary importance in various applied situations like airplane icing [1], thermal spray coating of surfaces [2], soldering [3], or three-dimensional additive manufacturing [4-6]. It is also encountered in geophysics, like in the flow of molten lava [7] or the formation of ice stalagmites[8-10] or snow penitentes [11].

The spreading of liquids on substrates is essentially ruled by an equilibrium between capillarity and viscous stress near the triple line, where solid, liquid, and vapor meet [12]. When solidification is induced from a cold substrate, one has to combine hydrodynamics of spreading with heat transfer, phase change, and solidification front dynamics in a complex, singular, and dynamically evolving geometry. One of the main difficulties to overcome is the regularization of a singularity at the triple line in both the viscous shear stress [12-14] and the thermal heat flux [15,16].

In particular, the forming solid can induce a pinning force in the vicinity of the triple line, which can lead to discontinuous spreading or spreading arrest. Then the natural question that arises is to determine this arrest condition. However, studies of contact line dynamics in the presence of solidification are scarce. Some studies have attempted to address this issue, but the answer remains elusive. Among the pioneers, Schiaffino and Sonin studied experimentally and theoretically the spreading of liquid wax on a growing basal solid wax [16-18]. The liquid puddle was fed by a continuous spraying of droplets. They proposed a pinning criterion based on a critical value for the apparent contact angle. However, their criterion is very specific to the case of a liquid puddle 
TABLE I. Physical properties of hexadecane and pentadecane in the liquid and solid states. Extracted from Refs. [22,23].

\begin{tabular}{lcccc}
\hline \hline & $\begin{array}{c}\mathrm{C}_{16} \mathrm{H}_{34} \\
\text { (liquid) }\end{array}$ & $\begin{array}{c}\mathrm{C}_{16} \mathrm{H}_{34} \\
\text { (solid) }\end{array}$ & $\begin{array}{c}\mathrm{C}_{15} \mathrm{H}_{32} \\
\text { (liquid) }\end{array}$ & $\begin{array}{c}\mathrm{C}_{15} \mathrm{H}_{32} \\
\text { (solid) }\end{array}$ \\
\hline$\mu(\mathrm{Pas})$ & 0.003 & - & 0.003 & - \\
$\rho_{m}\left(\mathrm{~kg} / \mathrm{m}^{3}\right)$ & 774 & 833 & 774 & 786 \\
$\gamma(\mathrm{N} / \mathrm{m})$ & 0.028 & - & 0.026 & - \\
$C_{p}[-\mathrm{J} /(\mathrm{kg} \mathrm{K})]$ & 2310 & 1800 & 2200 & 2170 \\
$\alpha\left(\mathrm{m}^{2} / \mathrm{s}\right)$ & $8.4 \times 10^{-8}$ & $1.0 \times 10^{-7}$ & $1.05 \times 10^{-7}$ & $1.05 \times 10^{-7}$ \\
$k[\mathrm{~W} /(\mathrm{m} \mathrm{K})]$ & 0.15 & 0.15 & 0.18 & 0.18 \\
$\mathcal{L}(\mathrm{J} / \mathrm{kg})$ & $2.3 \times 10^{5}$ & $2.3 \times 10^{5}$ & $1.6 \times 10^{5}$ & $1.6 \times 10^{5}$ \\
\hline \hline
\end{tabular}

advancing on its own frozen basal melt, with a quadruple line. More recently, Tavakoli et al. [19] investigated the spreading of a droplet on cold glass substrate. From the fact that the conductivity of ambient air is negligible compared to that of liquid, the isotherm $T=T_{m}$ is normal to the free surface, and the heat flux is assumed to diverge (or at least to be strongly enhanced) when approaching the triple line. Hence they proposed a condition of pinning based on a critical frozen volume at the triple line. Finally, de Ruiter et al. [20] studied experimentally the early spreading and arrest of a drop of hexadecane spreading on cold substrates. The experiments were consistent with an arrest criterion based on a critical contact-line velocity for pinning, which value is dependent on $\Delta T=T_{m}-T_{s}$, suggesting a mechanism ruled by kinetic undercooling [21].

The present study aims to determine experimentally the dynamics of liquid spreading in the presence of solidification induced by a cold substrate. The main question we intend to answer is how and when the local formation of a solid (presumably in the vicinity of the substrate, and near the contact line) can induce the liquid pinning and arrest of spreading? We opted for an original experimental setup that allows to operate under situations of steady states, where the substrate temperature and the spreading velocity are accurately controlled. We visualize and record the contact-line dynamics and the solidification front. We observe that the spreading dynamics can adopt two distinct behaviors: (1) continuous advancing of the contact line at constant velocity and (2) discontinuous advancing with stick-slip dynamics. The transition between the two regimes is determined by a threshold in velocity that is temperature-dependent. Beyond the measurement of this threshold, we also carried out measurements in the stick-slip regime to determine the typical frequency of the stop-and-go dynamics as well as the contact angle at the onset of depinning.

Finally, since the pinning transition offers a way to measure a solidifying front velocity in this peculiar geometry of liquid spreading, we compare our results to direct measurements of the front dynamics in a bulk, boundless geometry, which reveals a crucial influence of the front stability.

The paper is organized as follow: in Sec. II we describe the experimental setup and show qualitative observations, in Sec. III we present the experimental results, and in Sec. IV we discuss the criterion for pinning in light of independent measurements of front velocity versus temperature. Section V concludes with the main outcomes of our results.

\section{EXPERIMENTAL SETUP}

A liquid droplet is deposited on a moving substrate, a 4-inch-diameter polished silicon wafer, of temperature $T_{s}$ below the melting point of liquid $T_{m}$. In order to circumvent the difficulties inherent to water (operating below $0{ }^{\circ} \mathrm{C}$ ), we opted for linear alkane, namely, pentadecane $\left(\mathrm{C}_{15} \mathrm{H}_{32}\right)$ and hexadecane $\left(\mathrm{C}_{16} \mathrm{H}_{34}\right)$, as liquids (see properties in Table I, extracted from Refs. [22,23]). The choice for these liquids was dictated by several criteria. First, their melting temperature of $8.5^{\circ} \mathrm{C}$ and $18.5^{\circ} \mathrm{C}$, respectively, allows us to operate under ambient conditions. Furthermore, contrary to water, both of these liquids exhibit very reduced supercooling. Also, condensation on the substrate can be prevented by operating in a dry atmosphere. However, during our attempts with water at 


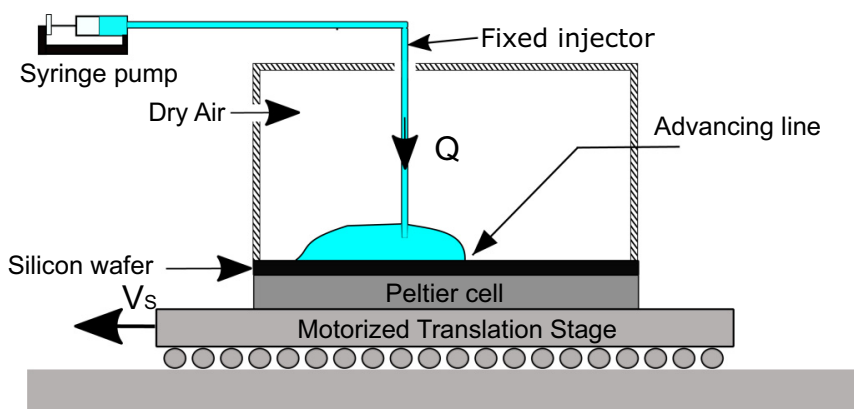

FIG. 1. Sketch of the experimental setup.

$T<0{ }^{\circ} \mathrm{C}$, we could observe significant evaporation that influences the dynamics. With our nonvolatile liquids (and still with viscosity within the range of usual liquids like water), this evaporation is nonsignificant, and we simply prevent the condensation of water from atmosphere on the substrate by injecting dry air inside the box in which the wafer is placed.

To operate in partial wetting situations with relatively low contact-angle hysteresis is a necessary condition to observe clear and reproducible dynamics. Hence, the substrate is chemically treated with a grafting of a self-assembled monolayer (SAM) of a low-energy fluorosilane: $1 \mathrm{H}, 1 \mathrm{H}, 2 \mathrm{H}$, $2 \mathrm{H}$ perfluorodecyltrichlorosilane, hereafter denoted as PFTS. This grafting with covalent bonds is known to ensure a robust and durable coating, both mechanically and chemically. When any sign of coating degradation would be detected, we would replace the wafer with a new coating. For both liquids, the resulting static (receding and advancing) wetting angles are $\theta_{r}=23^{\circ} \pm 3^{\circ}$ and $\theta_{a}=66^{\circ} \pm 2{ }^{\circ}$ and independent of the substrate temperature.

The liquid is poured through a needle injector of diameter $d=2 \mathrm{~mm}$, held at a distance of $2 \mathrm{~mm}$ from the substrate. The liquid is pumped by a syringe driver (Havard Apparatus reference 55-3333) at constant flow rate $Q$, forming a continuously filled capillary bridge between needle and substrate. The value of $Q$ is chosen within a certain range, depending on both $V_{s}$ and $\Delta T$, in order to ensure that the injector and the substrate remain connected by the liquid. If $Q$ is too small, the drop stretches and pinches off, and if $Q$ is too large, one does not observe any steady state.

The temperature of the wafer $T_{S}$ is imposed by a Peltier cell device and controlled with a feedback loop with an accuracy of $\pm 0.25^{\circ} \mathrm{C}$. The undercooling $\left(\Delta T=T_{m}-T_{s}\right)$ ranges from $0.25^{\circ} \mathrm{C}$ to $5^{\circ} \mathrm{C}$. To avoid external perturbations, the substrate and the injecting needle are enclosed in a transparent plexiglass cylinder. The temperature of liquid before injection, ambient air, and injector are kept at $21 \pm 1{ }^{\circ} \mathrm{C}$.

The wafer, Peltier cell, dry air injector, and transparent box are bound on a micro-positioning motorized stage (Newport Universal Motion Controller ESP 300). This allows us to control the velocity of the substrate within a range of 0.01 to $100 \mathrm{~mm} / \mathrm{s}$, as illustrated in Fig. 1. Conversely, the injecting needle is fixed to the laboratory frame. The top of the droplet (or bridge) remaining pinned on the needle, the substrate velocity is also the advancing velocity of the deposited liquid on the substrate. The side view of the drop shape and dynamics is recorded with a camera (IDS, ref. UI-306XCP-M), together with back-lighting from a fiber optic cold light.

In Table I we indicate the physical properties (surface tension $\gamma$, melting temperature $T_{m}$, latent heat $\mathcal{L}$, heat capacity $C_{p}$, density $\rho_{m}$, thermal diffusivity $\alpha$, thermal conductivity $k$, and viscosity $\mu$ ) of both alkane, in solid and liquid phases.

\section{OBSERVATIONS OF THE DYNAMICS}

The dynamics of spreading is investigated by prescribing the value of the undercooling $\Delta T$. We also operate with constant flow rate $Q$. Keeping these two quantities constant, we carry out different runs varying the velocity $V_{s}$ of the translation stage in the laboratory frame. For each run, we capture 

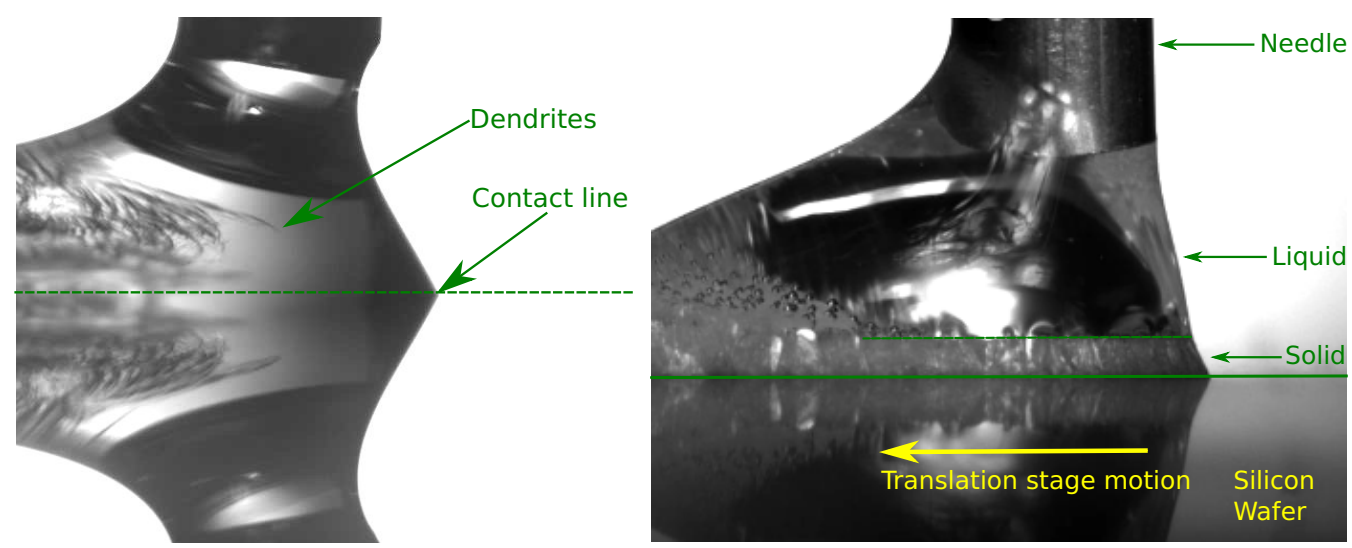

FIG. 2. Typical shape of the drop of hexadecane near the advancing line in the continuous regime (left: $\Delta T=0.25^{\circ} \mathrm{C}, V_{s}=0.8 \mathrm{~mm} / \mathrm{s}, Q=200 \mu \mathrm{l} / \mathrm{min}$ ) and in the stick-slip regime (right: $\Delta T=2{ }^{\circ} \mathrm{C}$, $V_{s}=1 \mathrm{~mm} / \mathrm{s}, Q=100 \mu \mathrm{l} / \mathrm{min}$ ). In the latter situation, the solid front catches up the advancing line. In both cases and in the next images, the substrate moves from right to left. The green line represents the substrate vertical position, below which the reflected image of the drop can be seen.

and record the whole dynamics of the advancing contact line, as well as the shape of the free surface and the solidification front. The substrate is carefully cleaned with ethanol and dried between two runs. Then we start a new series of runs with a different value of $\Delta T$.

For each value of $\Delta T$ within a given range, there exists a critical velocity for the plate where the motion of the contact line undergoes a transition from continuous to stop-and-go. For low undercooling $\Delta T$ and high enough velocity $V_{s}$, the advancing contact line dynamics is continuous and remains at the same location in the laboratory frame: the velocity of the contact line is $V=0$ in the laboratory frame. For strong undercooling $\Delta T$ and low enough velocity $V_{s}$, we observe discontinuous dynamics. This stick-slip motion coincides with the observation of the solidification front reaching the liquid wedge at the triple line. The velocity $V$ also takes nonconstant values, jumping from $V=0$ to $V=-V_{s}$ in the laboratory frame (the advancing velocity in the frame of the substrate being then $\left.V+V_{s}\right)$. The determination of the critical velocity $V_{c}(\Delta T)$ defining the transition is limited to small enough values of $\Delta T$, typically below $5{ }^{\circ} \mathrm{C}$, due to the maximal value prescribed by the translation stage (about $100 \mathrm{~mm} / \mathrm{s}$ ). Also, due to the limited size of the wafer, some experiments at high velocities were dismissed as it was obvious that the duration of the experiment was too short for the contact-line motion to reach a steady regime. In Fig. 2, we show the typical shapes of the drop in the continuous spreading (Left) and stick-slip (Right) regimes. In the latter situation, the freezing front reaches the advancing contact-line.

It is remarkable that the measured transition threshold is almost independent on the flow rate and remains constant with $Q$ over a large range from $\mathrm{Q}=50 \mu \mathrm{l} / \mathrm{min}$ to $\mathrm{Q}=40000 \mu \mathrm{l} / \mathrm{min}$.

Let us also notice that the stick-slip can either show a rather periodic motion or be rather aperiodic. In both cases, averaged characteristic values for the frequency and jump lengths can be extracted from long-time acquisitions.

The shape of the liquid free surface also exhibits two different behaviors depending on which regime is selected: while this shape remains steady in the continuous regime, it fluctuates significantly during the stick-slip regime. Thus the dynamical contact angle at the advancing wedge $\theta_{\text {ad }}$ also exhibits sharp variations followed by a slower relaxation, and conversely $\theta_{\text {ad }}$ remains almost constant in the continuous regime.

In Fig. 3 we shows an example of side view of advancing contact line at relatively high velocity $V$ and small $\Delta T$. In this situation, the liquid slides along the substrate and we observe the solidification front behind the liquid contact line. The sequence shows a transient behavior over a few seconds after the plate is put in motion and before the liquid shape and solid front reach a final state. By 

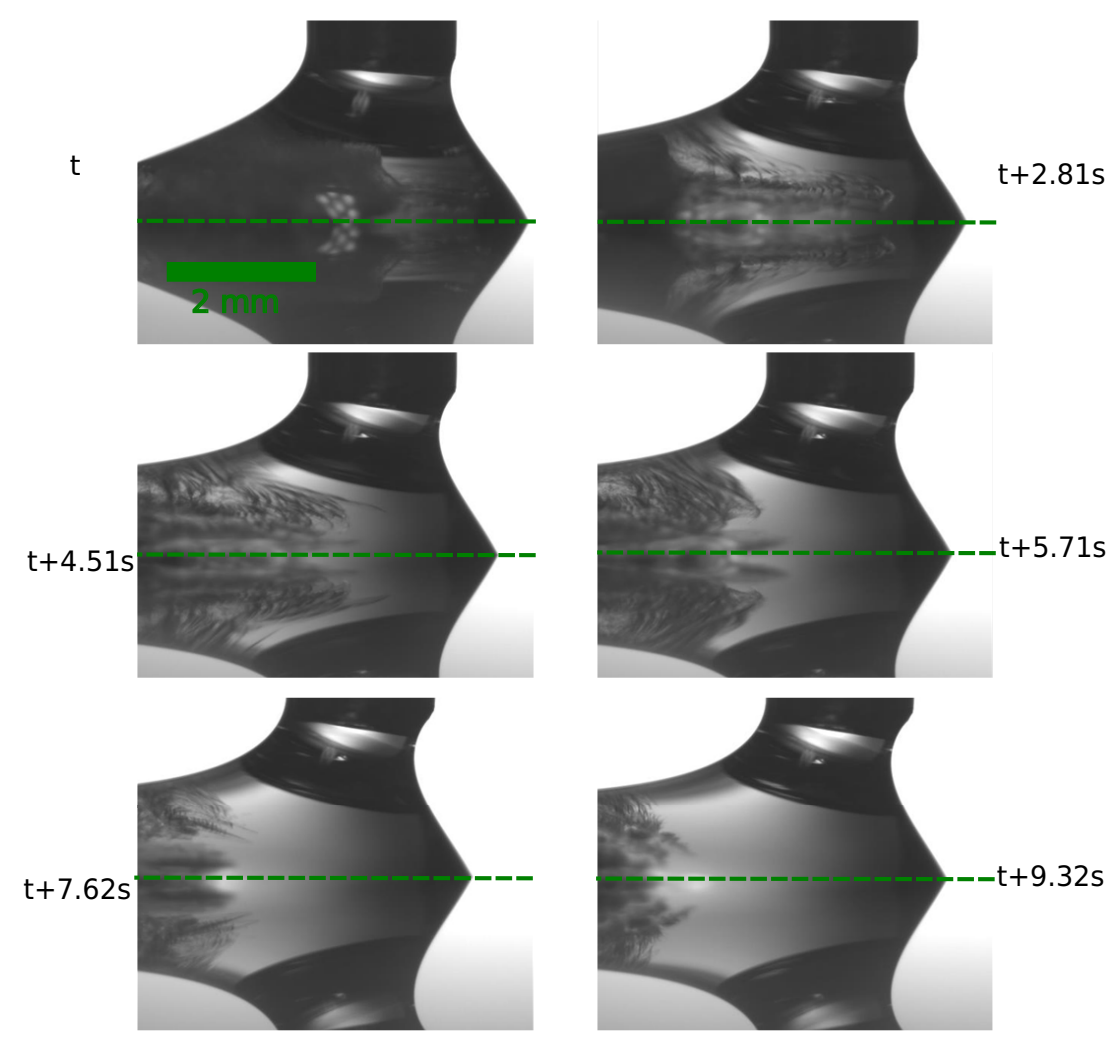

FIG. 3. Sequence of transient behavior of a drop experiencing continuous advance on a cold moving substrate $\left(\Delta T=0.25^{\circ} \mathrm{C}, V_{s}=0.8 \mathrm{~mm} / \mathrm{s}, Q=200 \mu \mathrm{l} / \mathrm{min}\right)$. The solid-liquid front visibly recedes away from the advancing line. The drop shape and contact-line location reach a steady state. The corresponding typical video is available in Ref. [24].

looking within the liquid, one clearly sees that during these few seconds, the solidification front recedes away from the contact line.

Let us describe the typical situation of continuous dynamics: after a few seconds of a transient behavior, the position of the contact line stabilizes at a position $X \simeq X_{0}$ in the laboratory frame. Also, after a short transient, the contact angle remains constant throughout the experiment. Unavoidable tiny fluctuations due to substrate imperfections are observed.

If now the substrate velocity is decreased below a critical value $V_{s}<V_{c}$, keeping $\Delta T$ constant, we observe a stick-slip dynamics, and the transient behavior is now illustrated by a typical sequence like that in Fig. 4. Clearly the solid front, initially behind the contact line, catches up and induces the pinning of the contact line for some time. As the plate continues to move, the liquid eventually unpins from the solid front and experiences a phase of sharp acceleration (during which $V \gg V_{s}$ ). The solidification disturbs the liquid spreading and changes the dynamics of contact line on the substrate.

The typical duration between each slip event ranges between 1 and $15 \mathrm{~s}$, depending on the experimental conditions. The duration of the stick phase is generally longer than that of the slip one. Figure 5 shows the typical behavior of both the contact-line position and the advancing dynamical contact angle $\theta_{\mathrm{ad}}$, in the stick-slip regime.

\section{QUANTITATIVE RESULTS}

\section{A. The threshold for stick-slip spreading}

We measure the threshold velocity $V_{c}$ for different $\Delta T$, by operating at fixed $\Delta T$, starting from a continuous spreading regime and slowly decreasing $V_{s}$. The value of $V_{c}$ is retained as soon as the 

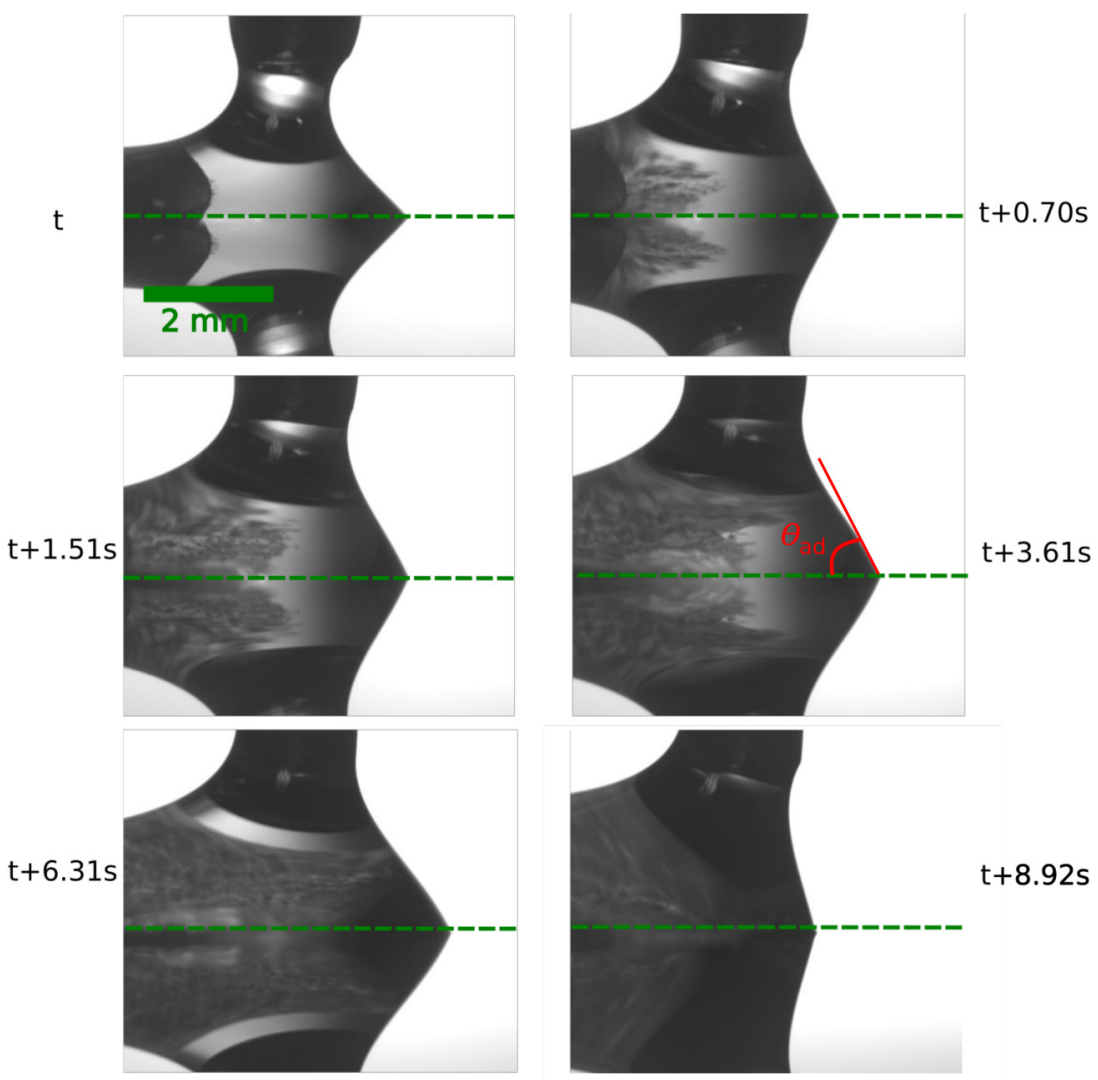

FIG. 4. Sequence of transient behavior of a drop experiencing discontinuous (stick-slip) dynamics on a cold moving substrate $\left(\Delta T=0.75^{\circ} \mathrm{C}, V_{s}=0.5 \mathrm{~mm} / \mathrm{s}, Q=500 \mu \mathrm{l} / \mathrm{min}\right)$. The solid-liquid front visibly catches up to the advancing line before pinning occurs, leading to an arrest of the contact line and an increase of the advancing angle. The corresponding typical video is available in Ref. [24].

first occurrence of advancing contact-line pinning is recorded. We also control that this pinning is not due to any dust on the substrate, and hence that it also corresponds to a situation where the solid lies in the vicinity of the contact line.

Figure 6 shows this critical velocity $V_{c}$ versus undercooling $\Delta T$, for both hexadecane (red squares) and pentadecane (blue squares). For the two liquids studied, the critical velocity follows a power law with the best fit on the exponent yields

$$
V_{c}=a \times \Delta T^{2.65} .
$$

Also plotted in Fig. 6 are the values of the maximal velocity of the solid front measured with an independent setup described later (red and blue circles). The error bars reflect the uncertainty on the substrate temperature, which cannot be controlled better than $\pm 0.25^{\circ}$. The prefactor $a$ equals 1.075 for pentadecane and 1.587 for hexadecane.

\section{B. Instantaneous velocity and critical angle}

We now focus closer on the stick-slip dynamics. First, we record the relative velocity between contact line and substrate versus time during several stick-slip events. A typical example is shown in Fig. 7, where the value of velocity $\langle V\rangle$ averaged over seven stick-slip events is plotted versus $\left(t-t_{0}\right)$, the time after each onset of slip event and $t_{0}$ taken as the initial time of each slip event. We 


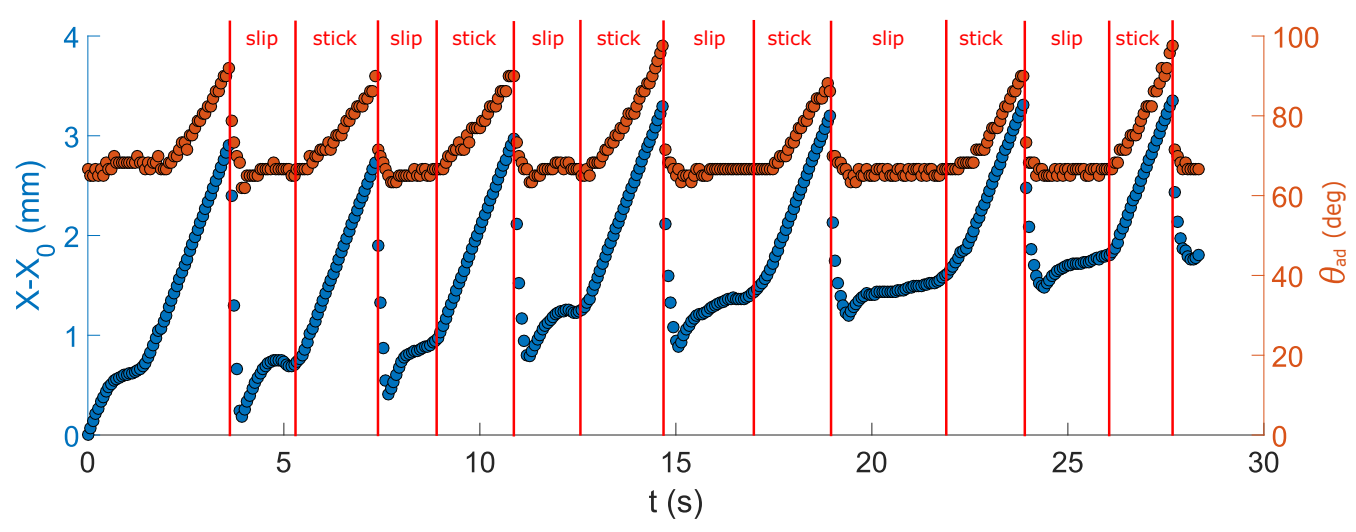

FIG. 5. Advancing dynamical contact angle $\theta_{\text {ad }}$ and relative position of contact line $\left(X-X_{0}\right)$ versus time, of hexadecane, over several stick-slip events $\left(\Delta T=0.75^{\circ} \mathrm{C}, V_{s}=1 \mathrm{~mm} / \mathrm{s}, Q=1100 \mu \mathrm{l} / \mathrm{min}\right)$.

define the stick phase, respectively, by the moment the contact-line velocity $\mathrm{V}$ (or its average $\langle V\rangle$ ) equals $-V_{s}$. It is indicated by arrows in the plot. We observed experimentally that $\langle V\rangle$ overcomes the prescribed substrate velocity $V_{s}$ (here, $1 \mathrm{~mm} / \mathrm{s}$ ) by one and a half order of magnitude. Furthermore, the duration of the slip phase (about half a second in the example shown) is generally much shorter than that of the stick phase (here about $3 \mathrm{~s}$ ). In the example shown, a bump in velocity appears about $0.7 \mathrm{~s}$ after the initial time $t_{0}$, which suggests that the pinning can sometimes appear in two stages.

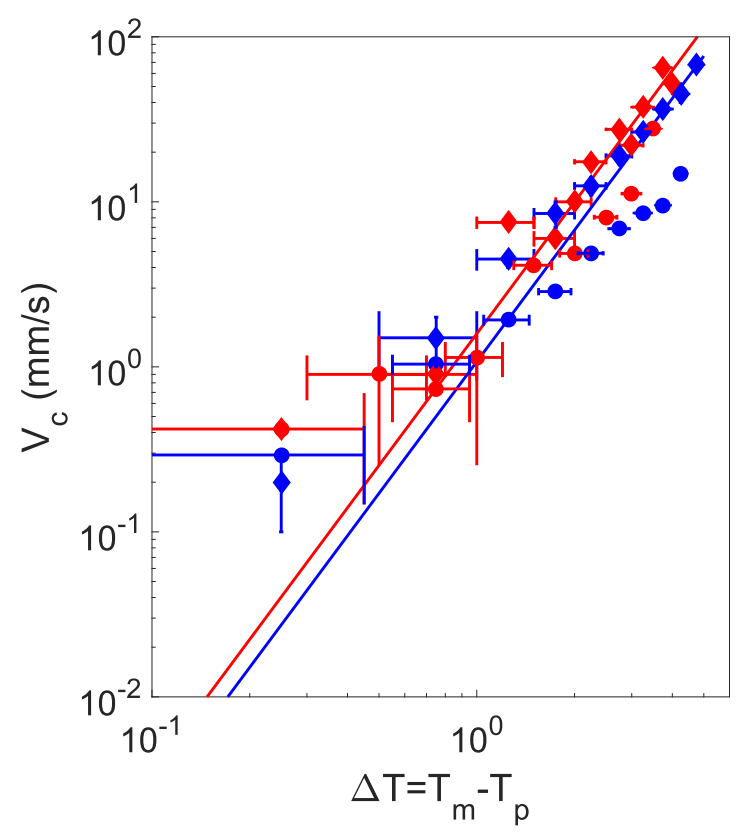

FIG. 6. Critical velocity $V_{c}$ versus $\Delta T$ for pentadecane (blue diamonds) and hexadecane (red diamonds). The blue and red circles stand for the velocity of the solid front directly measured in a bulk experiment (setup in Fig. 10 detailed in Sec. V), respectively, for pentadecane and hexadecane. The red and blue plain lines represent the best fit by a power law $V_{c} \propto \Delta T^{2.65}$. The power law is also emphasized in the insert where the data are plotted in log-log axes. 


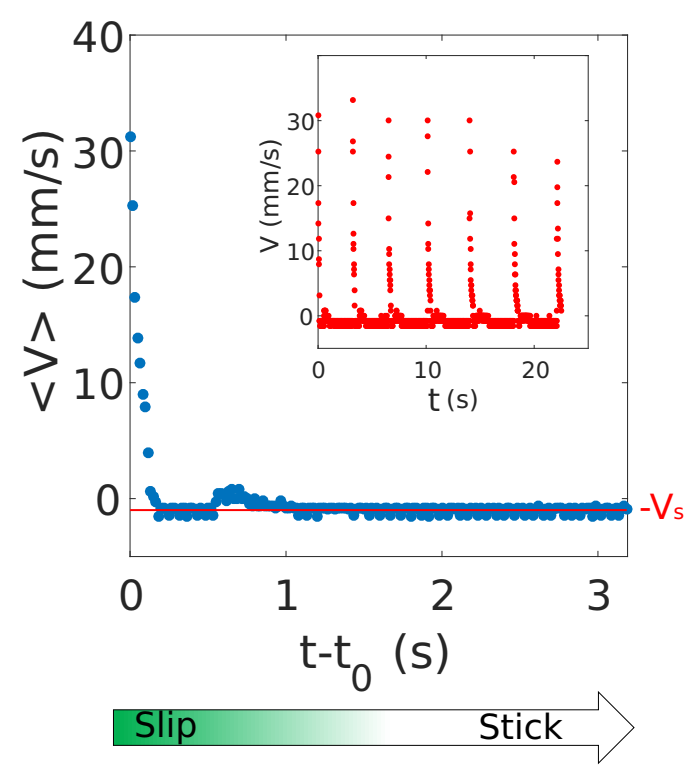

FIG. 7. Relative velocity of the advancing contact line versus time $\left(t-t_{0}\right)$, with $t_{0}$ is the time at the onset of sliding. The velocity is averaged from seven successive stick-slip events, which are represented in the insert $\left(\Delta T=0.75^{\circ} \mathrm{C}, V_{s}=1 \mathrm{~mm} / \mathrm{s}, Q=1100 \mu \mathrm{l} / \mathrm{min}\right)$. The arrow below shows the approximate time locations of the slip and stick phases, with the gradation of color indicating the relative uncertainty of the transition when considering the averaged velocity $\langle V\rangle$.

During the stick phase, the forming solid pins the liquid at its contact line while the translation stage continues moving. Hence, the apparent angle (i.e., formed between the liquid and the horizontal substrate) increases with time until it reaches a critical value; see Fig. 5. In the seek for a pinning criterion, we also recorded the critical angle $\theta_{c}$ (at the onset of depinning) beyond which the liquid unpins and spreads ahead the solid front. See Ref. [24] for the values of $\theta_{c}$ versus $V_{s}$ at different values of $\Delta T$ and two liquids, $\theta_{c}$, in ranges between $60^{\circ}$ and $105^{\circ}$. Although the trend roughly suggests an increase of the lower and upper bounds of $\theta_{c}$ with $V_{s}$, no noticeable dependency on the different quantities $\left(V_{s}, \Delta T, Q\right)$ could be deduced from these measurements. Also, it is to be noted that this critical angle could not be related to the maximal velocity measured and presented in Fig. 7. For instance, a critical angle of $90^{\circ}$ would lead to a contact-line velocity of roughly $150 \mathrm{~mm} / \mathrm{s}$ in the frame of the capillary-viscous Cox-Voinov theory [25]. We clearly measured far slower maximal velocities. It is not that surprising considering that at $V=30 \mathrm{~mm} / \mathrm{s}$ (the typical order of the maximal velocity measured under moderate $V_{s}$ and $\Delta T$ ), the Reynolds number is about 10. Therefore, inertia is not negligible in the spreading process (which will lead to major inertial slow down), and we are out of the framework of Cox-Voinov's theory.

\section{Influence of the injection flow rate}

We keep $Q$ within the range for the substrate and the injector to be connected (see Sec. II), and we check whether or not $Q$ influences the dynamics of stick-slip. First and probably most importantly, the flow rate $Q$ was found not to influence the threshold $V_{c}(\Delta T)$. This result suggests that the liquid injected at ambient temperature does not influence the heat transfer and the flow in the vicinity of the substrate where pinning occurs. Still, we investigate further by checking if $Q$ could influence the dynamics of the stick-slip regime. By carefully recording and extracting dynamics at different $Q$, it turns out that the stick-slip dynamics does not exhibit significant change. We extracted an averaged frequency of the stick-slip, which is basically the inverse of the time between 


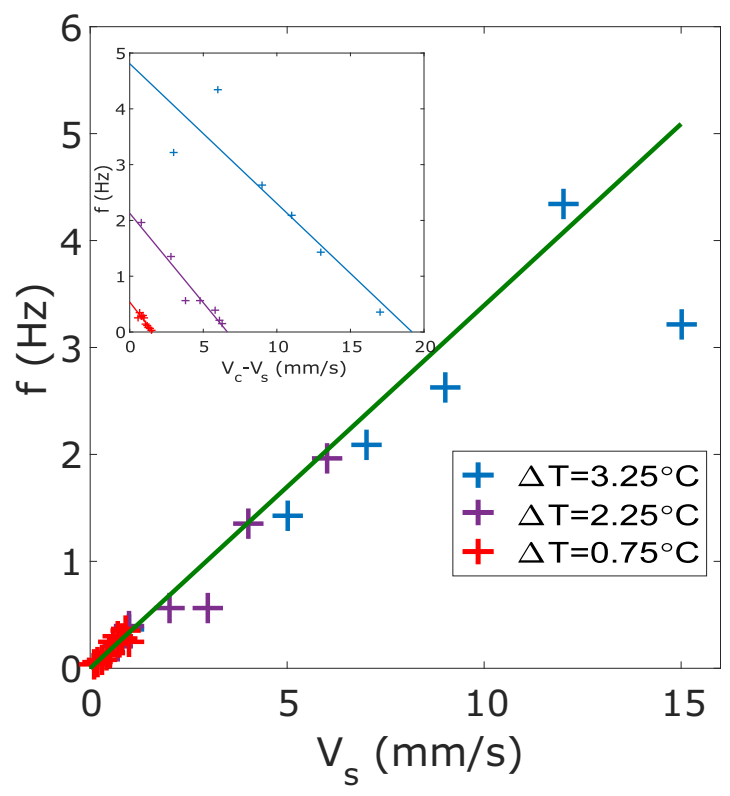

FIG. 8. Frequency of stick-slip versus substrate velocity $V_{s}$ for various $\Delta T=0.75,2.25$, and $3.25^{\circ} \mathrm{C}$. Insert: Frequency versus velocity difference with respect to threshold $\left(V_{c}-V_{s}\right)$. The plain lines result from the best linear fit of the data.

two onsets of slip events. The frequency barely changes with $Q$ but increases with $V_{s}$ See Ref. [24] for frequency versus flow rate $Q$ (for three different velocities and the same $\Delta T$ ). This confirms that $Q$ is a parameter of secondary importance in the experiments, and hence we now investigate the influence of $V_{s}$.

\section{Frequency of stick-slip}

A typical dependence of the frequency of stick-slip on $V_{s}$ is shown in Fig. 8 for three values of $\Delta T$ and versus $V_{c}-V_{s}$ in the insert. The frequency is averaged during a full sequence of stick-slip events, after the dynamics reached a permanent steady state, over a typical distance of $40 \mathrm{~mm}$ along the substrate. The range of velocities is between the onset of stick-slip at fixed $\Delta T$ to roughly 10 times slower, hence far from the threshold. The increase of $f$ with $V_{s}$ follows a fairly linear trend, which suggests that the typical length of jump in the slip phase $l_{j}=\frac{V_{s}}{f}$ is roughly constant and rather independent on $\Delta T$. The best linear fit gives $l_{j}=3.3 \mathrm{~mm}$, hence a value close to, but slightly larger than, the capillary length $l_{c}=\sqrt{\frac{\gamma}{\rho g}}$, which equals $1.85 \mathrm{~mm}$ and $1.92 \mathrm{~mm}$ respectively for pentadecane and hexadecane.

Additionally, we carried out experiments varying together $\Delta T$ and $V_{s}$, in order to keep as close as possible to the onset of stick-slip. Figure 9 shows the values of $f$ versus $V_{s}$. The linear dependence between $f$ and $V_{s}$ is confirmed and holds even very close to the threshold. Furthermore, the value of the coefficient relating $f$ and $V_{c}$ is a property of both the liquid and the substrate (roughly equal to $0.13 \mathrm{~mm}^{-1}$ for hexadecane on PFTS). This value, which is independent on $\Delta T$, suggests that the typical length of the jump at threshold during the stick-slip $\left(l_{j}^{*}=7.7 \mathrm{~mm}\right)$ remains finite and does not diverge. Therefore, since the continuous regime would be characterized by a jump length equal to infinity, the transition between continuous and stick-slip regime appears to be of first order (discontinuous). 


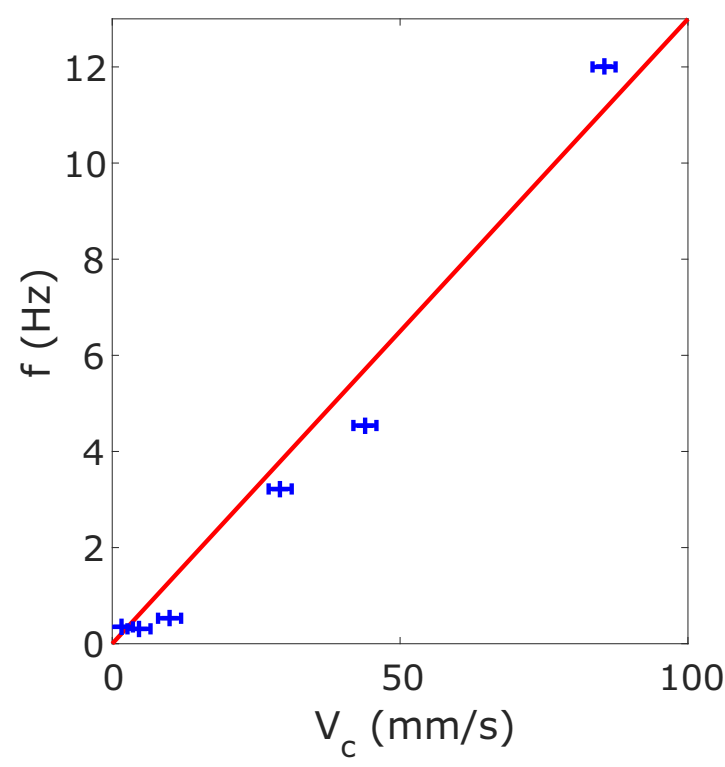

FIG. 9. Frequency of stick-slip close to the threshold of continuous dynamics versus $V_{c}(\Delta T)$.

\section{INTERPRETATION AND KINETIC CRITERION}

Recently, de Ruiter et al. [20] investigated the first steps of the axisymmetric spreading of a hexadecane drop on a substrate colder than the freezing temperature. By measuring the whole spreading dynamics and the final radius and angle of arrest versus substrate temperature, they found that their results were consistent with a kinetic criterion, i.e., that pinning would occur as soon as the spreading velocity (decreasing with time) would be smaller than the velocity of the solid-liquid front at a given temperature, here that of the substrate. They deduced that the speed of the front $V_{f}$ equals a power-law function of $\Delta T$ :

$$
V_{f}=\kappa(\Delta T)^{b}
$$

with $b=1$ giving the best fit.

In the present results, the fact that the stick-slip transition depends directly on $V_{s}$ suggests that a similar phenomenon could be at play here. In both studies, liquids are the same or similar, and the range of $\Delta T$ here is $0.5^{\circ} \mathrm{C}$ to $5^{\circ} \mathrm{C}$, whereas it is $0.5^{\circ} \mathrm{C}$ to $14^{\circ} \mathrm{C}$ in the study of de Ruiter et al. [20] From their results, we can deduce the critical contact-line velocity when pinning occurs, which would be analogous to our critical velocity $V_{c}$ : the arrest velocity followed an empirical relationship $V_{f}=\kappa \Delta T$, with $\kappa=0.011 \mathrm{~m} / \mathrm{s} / \mathrm{K}$. This yields an arrest velocity (and $V_{f}$ ) equal, respectively, to $0.0055 \mathrm{~m} / \mathrm{s}$ and $0.055 \mathrm{~m} / \mathrm{s}$ for $\Delta T=0.5^{\circ} \mathrm{C}$ and $5{ }^{\circ} \mathrm{C}$, which are values comparable (in order of magnitude) to ours under the same $\Delta T$. Still, our measurements (Fig. 6) yield $b=2.65$, hence significantly different from the trend found in de Ruiter et al. [20].

To check the dependence of the front velocity on $\Delta T$, we carry out independent measurements of $V_{f}$ in a different geometry, without the complexity of contact-line hydrodynamics, and where the front can be more directly observed. The setup is sketched in Fig. 10 (top): a cylindrical vessel containing pentadecane or hexadecane is cooled from its base, at fixed temperature $T_{s}<T_{m}$. The base is made of a wafer of silicon of the same nature as that of the translation stage setup. The birth and propagation of the solidification front is recorded by a camera acquiring sequences from above. As the depth of field is much smaller than the container size, and significantly smaller than the liquid height, we can record the solidification velocity in a plane parallel to the wafer. A typical sequence is shown in Fig. 10 (bottom). 


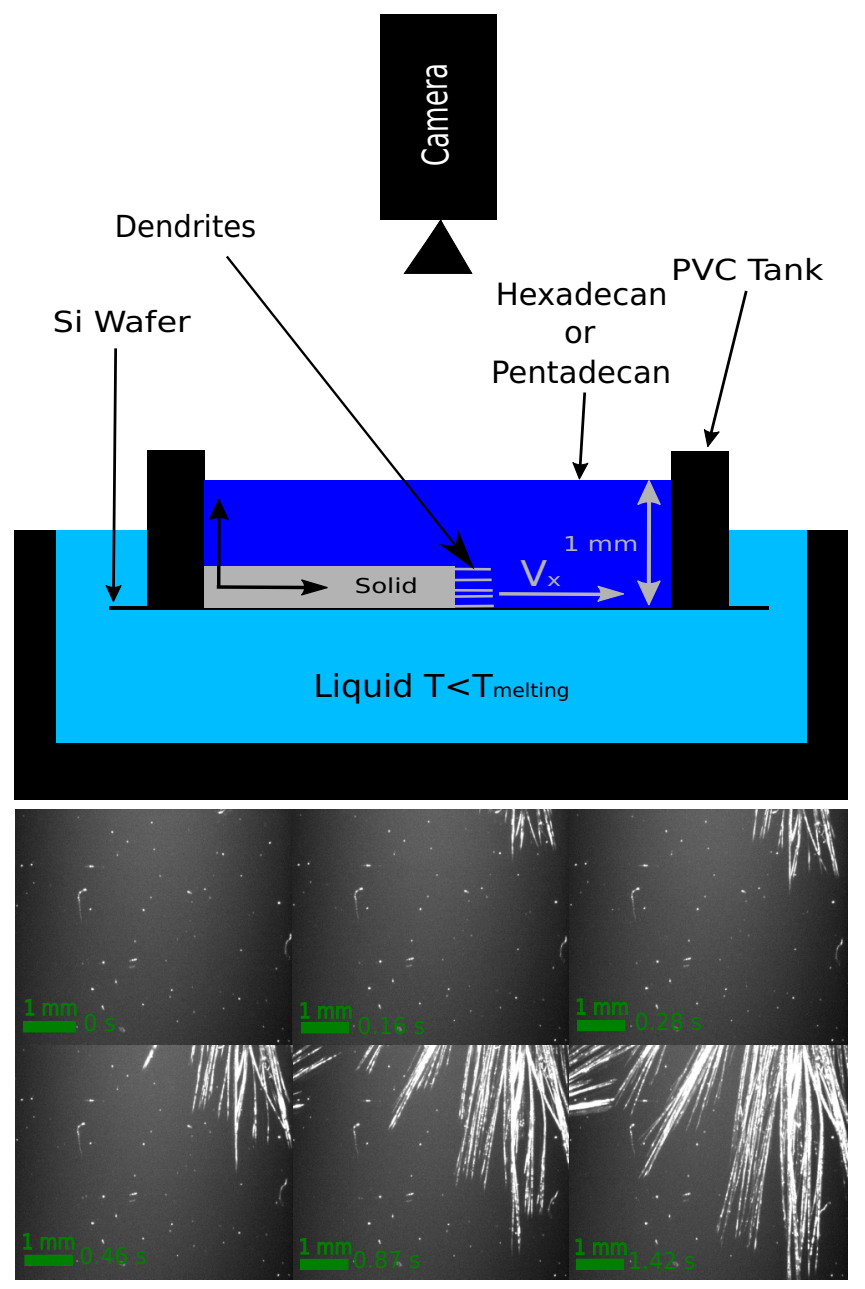

FIG. 10. (Top) Sketch of the bulk solidification setup allowing for the direct observation of the dynamics of the front. (Bottom) A typical sequence of the solidification front progression, showing dendritic shape with needles $\left(\Delta T=1.5^{\circ} \mathrm{C}\right.$, hexadecane).

The front is by no means flat and appears in the shape of dendritic needles, which structure is reminiscent from those of Figs. 2, 3, and 4. Measurements of velocity at the tip of needles is feasible by extracting the gray levels along their direction of propagation. From a significant set of measurements, we extracted the maximal value of this tip velocity for different values of $\Delta T$ from $0.25^{\circ} \mathrm{C}$ to $5^{\circ} \mathrm{C}$. The results are plotted in Fig. 6 (blue and red disks) and directly compared to those obtained from the translation stage experiments. It turns out that the dependence of $V_{f}$ on $\Delta T$ is consistent, at least in trend and order of magnitude, with $V_{c}$, although it shows a slight departure from a power law with exponent 2.65 .

Still, the relatively fair agreement between $V_{c}$ and $V_{f}$ versus $\Delta T$ reinforces the interpretation in terms of a kinetic criterion, i.e., that the transition from continuous to stick-slip dynamics is ruled by the velocity of the freezing front at the considered temperature. How to explain the quantitative discrepancy between the results of de Ruiter et al. [20] and the present ones? Actually, while those authors studied freezing-induced pinning, that occurs less than $1 \mathrm{~s}$ after liquid touchdown and most often in inertia-capillary-driven spreading, our experimental setup drives an advancing contact line 
toward a permanent steady regime (either continuous or discontinuous) within several seconds (see again the typical dynamics in Fig. 5). During this period of time (say, roughly $\tau=10 \mathrm{~s}$ or longer), heat diffusion takes place in the liquid in a typical layer of thickness $l_{T}=\sqrt{\alpha \tau}$ roughly equal to $1 \mathrm{~mm}$. Together with the shear-induced flow near the liquid-substrate interface, it can generate complex temperature gradient within the drop that, in turn, favors the Mullin-Sekerka instability [26] and the dendrites, as the latter constitute strongly nonlinear stages of this instability. Conversely in the short-time spreading experiments of de Ruiter et al., no such dendrites were observed prior to pinning and solidification rather appears within the form of circular growing spots [27].

The tip velocity of dendrites versus undercooling temperature was investigated experimentally by Glicksman and co-authors [28-30] and Davis [21]. An exponent $b$ slightly smaller or equal to 2.65 was found. From Figs. 3, 4, and 10, it is clear that the formation and propagation of such dendrites is consistent with the timescale of our experiments. Therefore, since our direct measurements of the tip velocity are consistent with Glicksman's and since the critical velocity $V_{c}$ versus $\Delta T$, although being an indirect measurement of the front velocity, follows a similar power law (Fig. 6), we propose the kinetic criterion as a plausible mechanism for the transition.

\section{CONCLUSIONS}

The spreading of a liquid on a substrate colder than freezing temperature, at imposed velocity, can show continuous or discontinuous dynamics. We characterized and measured the transition between these two regimes for two liquids (hexadecane and pentadecane) in partial wetting conditions and relatively low hysteresis with the substrate. The transition between continuous and stick-slip dynamics appears below a critical imposed velocity $V_{c}$, which value depends on $\Delta T=T_{m}-T_{s}$, the difference between freezing and substrate temperatures. The sequence of stick-slip consists in a pinning phase, followed by a sudden release of the contact line where the velocity can overcome the driving velocity by a factor of 50 . The release of the contact line occurs when the capillary force gets stronger than the pinning one, and the critical angle takes values in a rather large range, suggesting that the pinning force can fluctuate from a pinning event to another. What governs the strength of pinning to the solid front, showing a rather complex shape, remains unclear and should be the scope of further investigations. Typical measured critical velocities range from 0.1 to $100 \mathrm{~mm} / \mathrm{s}$, under a temperature range $\Delta T$ of roughly $5^{\circ} \mathrm{C}$.

The relatively good agreement between $V_{c}(\Delta T)$ and the maximal velocity of dendrites measured independently, together with the direct observation of dendritic front catching up the drop advancing line in the stick-slip regime, is consistent with a mechanism for the transition that involves kinetic undercooling. This effect is reminiscent of what was measured in the early spreading and arrest of hexadecane drops [20], with a similar order of magnitude for arrest velocities under the same $\Delta T$, although the relationships between $V_{c}$ and $\Delta T$ follow distinct power laws in both cases. This discrepancy could be attributed to the morphological differences between solid fronts in both cases, as in Ref. [20] no dendrites could be observed, and it is likely that the front is isotropic.

Our experiments also suggest that the transition is discontinuous, as the stick-slip frequency and jump length remain finite approaching the threshold. Finally, the injection flow rate does not influence significantly the transition and the stick-slip frequency.

Critical velocities measured at the onset of pinning remain far below the velocities involved in many applications mentioned in the introduction. On the one hand, it means that more prompt and violent mechanisms could be at play in some applied situations, especially those involving water drop impact. Clearly, the scope of our results does not encompass such situations, but, rather, is relevant in relatively slow liquid spreading where viscosity and capillarity balance each other. On the other hand, the range of critical velocities brings encouraging prospectives in the use of a dynamical way to delay and prevent the solidification of significant volumes of sessile liquids (including water), by wiping drops at high enough velocity.

Therefore, this work is a first step to understand what rules the spreading of a contact line on a cold substrate, where local solidification can lead to transient or permanent pinning. Our results 
have been obtained with n-alkane liquids. Such liquids present low heat conductivity and low supercooling. Future work must continue on other liquids such as water or metals in order to check whether or not the mechanisms are similar.

\section{ACKNOWLEDGMENTS}

We would like to thank Prof. H. P. Kavehpour for fruitful discussions. We are grateful for the technical support from A. Grados. We acknowledge the financial support by the Agence Nationale de la Recherche (ICEWET Project : ANR-15-CE08-0031).

[1] See, for example, SKYbrary, In-flight icing, http://www.skybrary.aero/index.php/In-Flight_Icing.

[2] P. Fauchais, M. Fukumoto, A. Vardelle, and M. Vardelle, Knowledge concerning splat formation: An invited review, J. Thermal Spray Technol. 13, 337 (2004).

[3] D. Attinger, Z. Zhao, and D. Poulikakos, An experimental study of molten microdroplet surface deposition and solidification: Transient behavior and wetting angle dynamics, J. Heat Transfer 122, 544 (2000).

[4] F. Gao and A. A. Sonin, Precise deposition of molten microdrops: The physics of digital microfabrication, Proc. R. Soc. Lond. A 444, 533 (1994).

[5] C. Ainsley, N. Reis, and B. Derby, Freeform fabrication by controlled droplet deposition of powder filled melts, J. Mat. Sci. 37, 3155 (2002).

[6] M. Vaezi, H. Seitz, and S. F. Yang, A review on 3D micro-additive manufacturing technologies, Int. J. Adv. Manu. Technol. 67, 1721 (2013).

[7] R. W. Griffiths, The dynamics of lava flows, Annu. Rev. Fluid Mech. 32, 477 (2000).

[8] M. B. Short, J. C. Baygents, and R. E. Goldstein, A free-boundary theory for the shape of the ideal dripping icicle, Phys. Fluids 18, 083101 (2006).

[9] A. Szu-Han Chen and S. W. Morris, Experiments on the morphology of icicles, Phys. Rev. E 83, 026307 (2011).

[10] A. Szu-Han Chen and S. W. Morris, On the origin and evolution of icicle ripples, New J. Phys 15, 103012 (2013).

[11] P. Claudin, H. Jarry, G. Vignoles, M. Plapp, and B. Andreotti, Physical processes causing the formation of penitentes, Phys. Rev. E 92, 033015 (2015).

[12] D. Bonn, J. Eggers, J. Indekeu, J. Meunier, and E. Rolley, Wetting and spreading, Rev. Mod. Phys. 81, 739 (2009).

[13] C. Huh and L. E. Scriven, Hydrodynamic model of steady movement of a solid/liquid/fluid contact line, J. Colloid Interface Sci. 35, 85 (1971).

[14] J. H. Snoeijer and B. Andreotti, Moving contact lines: Scales, regimes, and dynamical transitions, Annu. Rev. Fluid Mech. 45, 269 (2013).

[15] D. M. Anderson and S. H. Davis, Local fluid and heat flow near contact lines, J. Fluid Mech. 268, 231 (1994).

[16] S. Schiaffino and A. Sonin, Motion and arrest of a molten contact line on cold surface: An experimental study, Phys. Fluids 9, 2217 (1997).

[17] S. Schiaffino and A. Sonin, Molten droplet deposition and solidification at low Weber numbers, Phys. Fluids 9, 3172 (1997).

[18] S. Schiaffino and A. Sonin, On the theory for the arrest of an advancing molten contact line on cold solid of the same material, Phys. Fluids 9, 2227 (1997).

[19] F. Tavakoli, S. H. Davis, and H. P. Kavehpour, Contact line arrest in solidifying spreading drops, Langmuir 30, 10151 (2014).

[20] R. de Ruiter, P. Colinet, P. Brunet, J. H. Snoeijer, and H. Gelderblom, Contact line arrest in solidifying spreading drops, Phys. Rev. Fluids 2, 043602 (2017). 
[21] S. H. Davis, Theory of Solidification (Cambridge University Press, Cambridge, 2001).

[22] C. Vélez, M. Khayet, and J. M. Ortiz de Zárate, Temperature-dependent thermal properties of solid/liquid phase change even-numbered n-alkanes: n-Hexadecane, n-octadecane and n-eicosane, Appl. Energy 143, 383 (2015).

[23] C. Vélez, M. Khayet, and J. M. Ortiz de Zárate, Thermal properties of n-pentadecane, n-heptadecane and n-nonadecane in the solid/liquid phase change region, Int J. Therm. Sci. 94, 139 (2015).

[24] See Supplemental Material at http://link.aps.org/supplemental/10.1103/PhysRevFluids.4.033603 for the critical angle at the onset of sliding versus substrate velocity, for the frequency of stick-slip versus flow rate, and for the videos corresponding to sequences of Figs. 3 and 4.

[25] O. V. Voinov, Hydrodynamics of wetting, Fluid Dyn. 11, 714 (1976).

[26] J.-J. Xu, Unidirectional Solidification and Mullins-Sekerka Instability, in Interfacial Wave Theory of Pattern Formation, Springer Series in Synergetics Vol. 68 (Springer, Berlin, Heidelberg, 1998).

[27] J. H. Snoeijer (private communication).

[28] M. E. Glicksman, R. J. Schaefer, and J. D. Ayers, Dendritic growth—A test of theory, Metall. Trans. A 7, 1747 (1976).

[29] M. E. Glicksman, M. B. Koss, and E. A. Winsa, Dendritic Growth Velocities in Microgravity, Phys. Rev. Lett. 73, 573 (1994).

[30] M. E. Glicksman, Principles of Solidification: An Introduction to Modern Casting and Crystal Growth Concept (Springer-Verlag, New York, 2011). 